\title{
Blocking the development of postischemic cardiomyopathy with viral gene transfer of the apoptosis repressor with caspase recruitment domain
}

\author{
Subhasis Chatterjee, $\mathrm{MD}^{\mathrm{a}}$ \\ Lawrence T. Bish, BA ${ }^{\mathrm{b}}$ \\ Vasant Jayasankar, MD ${ }^{\mathrm{a}}$ \\ Allan S. Stewart, MD ${ }^{\mathrm{a}}$ \\ Y. Joseph Woo, MD \\ Michael T. Crow, $\mathrm{PhD}^{\mathrm{c}}$ \\ Timothy J. Gardner, MD \\ H. Lee Sweeney, $\mathrm{PhD}^{\mathrm{b}}$
}

From the Division of Cardiothoracic Sur-
gery a and Department of Physiology, ${ }^{\text {b Uni- }}$
versity of Pennsylvania School of Medi-
cine, Philadelphia, Pa, and the National
Institute on Aging, ${ }^{c}$ National Institutes of
Health, Baltimore, Md.

This work was supported by grants from the National Heart, Lung, and Blood Institute (HL07843 and HL59407).

Read at the Fifth Annual C. Walton Lillehei Resident Forum at the Eighty-second Annual Meeting of The American Association for Thoracic Surgery, Washington, DC, May 5-8, 2002.

Received for publication June 4, 2002; revisions requested Aug 19, 2002; revisions received Sept 10, 2002; accepted for publication Sept 24, 2002.

Address for reprints: Timothy J. Gardner, MD, Professor and Chief, Division of Cardiothoracic Surgery, University of Pennsylvania School of Medicine, 6 Silverstein Pavilion, 3400 Spruce St, Philadelphia, PA 19104 (E-mail: gardnert@uphs.upenn.edu).

J Thorac Cardiovasc Surg 2003;125:1461-9

Copyright $\odot 2003$ by The American Association for Thoracic Surgery

$0022-5223 / 2003 \$ 30.00+0$

doi:10.1016/S0022-5223(02)73229-7
Objectives: Apoptosis caused by acute ischemia and subsequent ventricular remodeling is implicated as a mediator of heart failure. This study was designed to assess the efficacy of in vivo viral gene transfer of the antiapoptotic factor apoptosis repressor with caspase recruitment domain to block apoptosis and preserve ventricular geometry and function.

Methods: In a rabbit model of regional ischemia followed by reperfusion, an experimental group treated with adenovirus-apoptosis repressor with caspase recruitment domain was compared with empty vector adenovirus-null controls. Cardiac function was assessed by echocardiography and sonomicrometry of the border zone compared with the normal left ventricle. Animals were killed at 6 weeks with measurements of ventricular geometry and apoptosis.

Results: Animals with the apoptosis repressor with caspase recruitment domain (ARC group) maintained higher ejection fractions at 4 and 6 weeks, and sonomicrometry demonstrated greater protection of border zone fractional shortening at 6 weeks compared with the control group. The ARC group maintained superior preservation of left ventricular geometry with less ventricular dilation and wall thinning. Finally, there was reduced apoptosis in the rabbits treated with apoptosis repressor with caspase recruitment domain compared with the controls.

Conclusions: Gene transfer of apoptosis repressor with caspase recruitment domain preserves left ventricular function after ischemia. The benefit at 6 weeks is postulated to result from an apoptosis repressor with caspase recruitment domainmediated reduction in apoptosis and ventricular remodeling. Adenovirus-apoptosis repressor with caspase recruitment domain administration offers a potential strategy after myocardial ischemia to protect the heart from late postischemic cardiomyopathy.

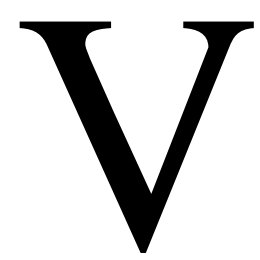

entricular injury after myocardial infarction can lead to progressive chamber enlargement and ventricular wall thinning. Wall thinning occurs in the infarcted region and the border zone (BZ) between the ischemic segment and the noninfarcted viable myocardium. ${ }^{1}$ The interaction of the area of infarction with remaining myocardium results in supraphysiologic stretch across the BZ, which augments the tension load. ${ }^{2}$ Because of the heightened wall tension, the ventricle remodels and undergoes dilation. With progressive ventricular dilation, 


\section{Extrinsic Pathway}

\section{Intrinsic Pathway}

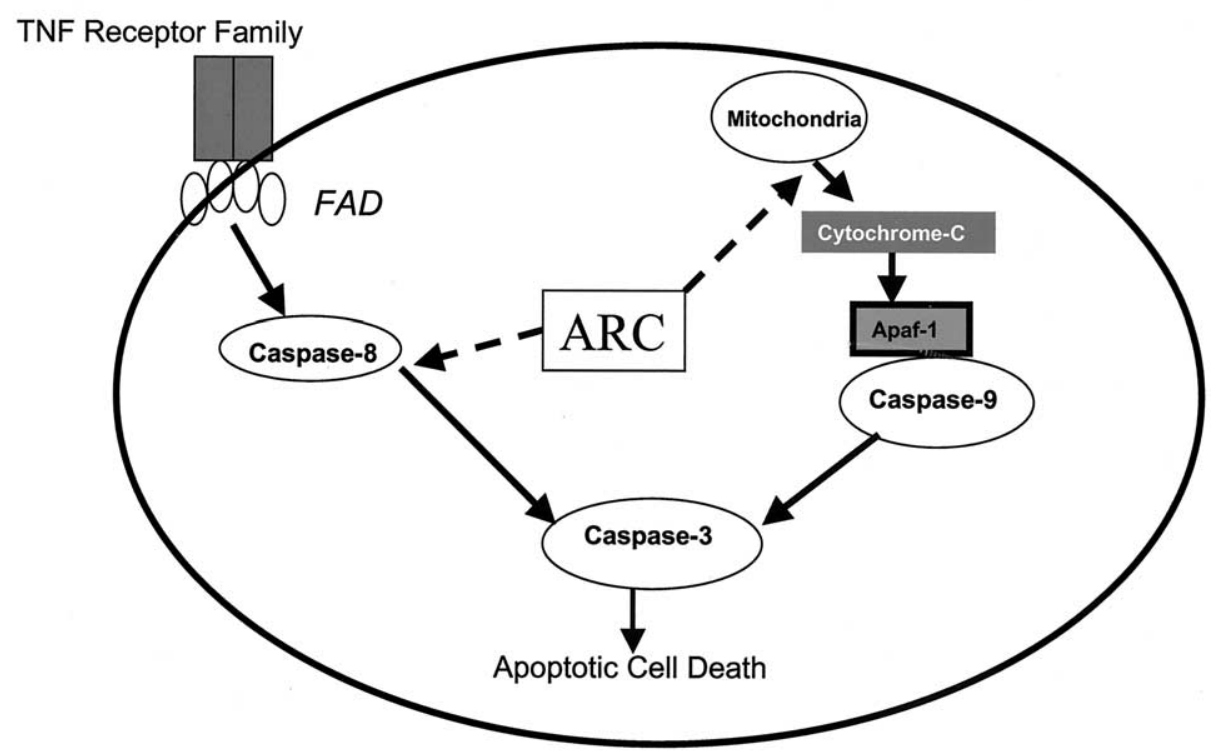

Figure 1. Pathways of apoptosis and site of action of ARC. Major pathways involved in the apoptotic cascade include extrinsic (death receptor pathway) and intrinsic (mitochondrial) pathway. ARC has been demonstrated to block the caspase 8 step of extrinsic pathway and release of cytochrome $c$ in the intrinsic pathway.

there is failure to maintain a compensatory proportional increase in wall thickness. Consequently, the wall thickness/ chamber radius ratio is not preserved, resulting in increased wall tension and decompensated eccentric ventricular hypertrophy and a decline in ventricular function. ${ }^{3}$

Although postischemic heart failure may have multiple causes, recent attention has been directed toward understanding the contribution of apoptosis or programmed cell death. Apoptosis is characterized by preservation of mitochondrial and sarcolemmal membranes, nuclear chromatin condensation, and phagocytosis by macrophages or neighboring cells without triggering an inflammatory response. It has been demonstrated in animal models and human patients after ischemia/reperfusion injury, myocardial infarction, congestive heart failure, and ischemic cardiomyopathy. ${ }^{4-6}$ It has recently been proposed that progressive ventricular dysfunction after infarction is the result of ongoing loss of cardiomyocytes from apoptosis. ${ }^{7}$

The activation of apoptosis is known to occur through 2

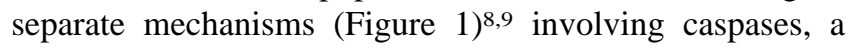
family of cysteine proteases that are synthesized as inactive precursors and proteolytically cleaved into their active form. ${ }^{10}$ The first mechanism has been termed the death receptor or extrinsic pathway. In this pathway, ligandbinding to a death receptor such as tumor necrosis factor receptor-1 recruits intracellular adaptor proteins such as tumor necrosis factor receptor-1-associated death domain and then Fas-associated death domain to bind procaspase-8, which is then cleaved into caspase- 8 .

The second major mechanism is the mitochondrial or intrinsic pathway. Mitochondria play a critical role in apoptosis, with the cytochrome c step in the electron transport chain the most vulnerable to disruption. ${ }^{11,12}$ When exposed to hypoxia and ischemia/reperfusion injury, mitochondria release cytochrome $\mathrm{c}$ into the cytosol to form a complex with the apoptosis activating factor (Apaf-1) and procaspase- $9 .{ }^{13}$ This complex is facilitated by a caspase-associated recruitment domain located on Apaf-1. Subsequently, the cytochrome c/Apaf-1/procaspase-9 complex activates caspase-9. Finally, both the extrinsic pathway involving caspase- 8 and the intrinsic pathway involving caspase-9 share a final common pathway and activate the downstream effector caspase-3, resulting in apoptotic cell death. ${ }^{8}$

An anti-apoptotic factor, the apoptosis repressor with caspase recruitment domain (ARC), demonstrates in vitro evidence of blocking both the intrinsic and extrinsic pathways (Figure 1). ARC is expressed almost exclusively in myogenic tissue and selectively interacts with the initiator caspases 2 and 8 attenuating death receptor-mediated induced apoptosis. ${ }^{14}$ It also blocks apoptosis associated with caspase-independent events such as hypoxia-induced cytochrome $\mathrm{c}$ release from the mitochondria. ${ }^{15}$ Finally, ARC can also prevent cellular changes associated with necrotic cell death by preventing mitochondrial dysfunction. ${ }^{16}$ 
In this study, we analyzed the effect of administering adenovirus-ARC (Adeno-ARC) after myocardial ischemia to determine its protective role in preventing the development of postischemic heart failure. After the initial ischemia/reperfusion injury, immediate cell death occurs in the infarcted zone by necrosis and apoptosis. As the ventricle remodels and the infarction proceeds to stiffen, we postulate that apoptosis persists at a low level in the BZ. Continued cell death and the loss of myocytes in this region may lead to ventricular dilatation and dysfunction. As a result, blocking apoptosis may reduce the drive toward continued ventricular dilation and preserve myocardial function. This would allow targeted gene therapy to be an adjunct to standard therapies after myocardial infarction in an effort to prevent subsequent postischemic heart failure.

\section{Methods}

\section{Adenoviral Vector Construction/Viral Delivery}

Replication-deficient adenoviral vector containing the transgene encoding human ARC with a constitutively active cytomegalovirus promoter was prepared as previously described. ${ }^{16}$ Empty vector Adeno-null virus ${ }^{17}$ (no transgene) with a cytomegalovirus promoter was constructed for controls. To demonstrate gene transfection using this method of viral delivery, an adenovirus construct encoding the reporter gene $\beta$-galactosidase ( $\beta$-Gal) was administered, and calculation of gene transfection was determined by scanning three sections through the BZ into Adobe Photoshop (Adobe Systems Incorporated, San Jose, Calif) (Figure 2).

\section{Animal Surgery}

All animals received humane care in compliance with institutional guidelines and the "Guide for the Care and Use of Laboratory Animals" prepared by the Institute of Laboratory Animal Resources, National Research Council, and published by the National Academy Press, revised 1996. Twenty-one New Zealand White rabbits (3-4 kg) were used. All rabbits were anesthetized with intramuscular doses of ketamine $(40 \mathrm{mg} / \mathrm{kg})$, xylazine $(2.5 \mathrm{mg} / \mathrm{kg})$, glycopyrrolate $(0.02 \mathrm{mg} / \mathrm{kg})$, and buprenorphine $(0.05 \mathrm{mg} / \mathrm{kg})$, orally intubated, and mechanically ventilated (Hallowell EMC model 2000, Pittsfield, Mass). A left thoracotomy was performed through the third intercostal space and a 6-0 polypropylene suture was placed around the circumflex artery halfway between the atrioventricular groove and the left ventricular (LV) apex. Both ends of the suture were placed through a red rubber catheter creating a tourniquet that was tightened to achieve complete cessation of flow as demonstrated by electrocardiographic changes and visual blanching. After 30 minutes, the tourniquet was released for reperfusion. The suture marking the site of occlusion remained facilitating identification of the $\mathrm{BZ}$ when the animal was put to death.

Next, the pulmonary artery (PA) and ascending aorta were each encircled with a $0-0$ silk suture and red rubber tourniquet. The PA tourniquet was tightened for complete PA occlusion, and the left atrium and ventricle allowed to empty for 5 seconds before the aortic tourniquet was tightened. A solution of $1000 \mu \mathrm{L}$ containing $5.0 \times 10^{10}$ particle-forming units of recombinant human AdCMV-ARC $(\mathrm{n}=8)$, suspended in $10 \%$ glycerol, was injected into
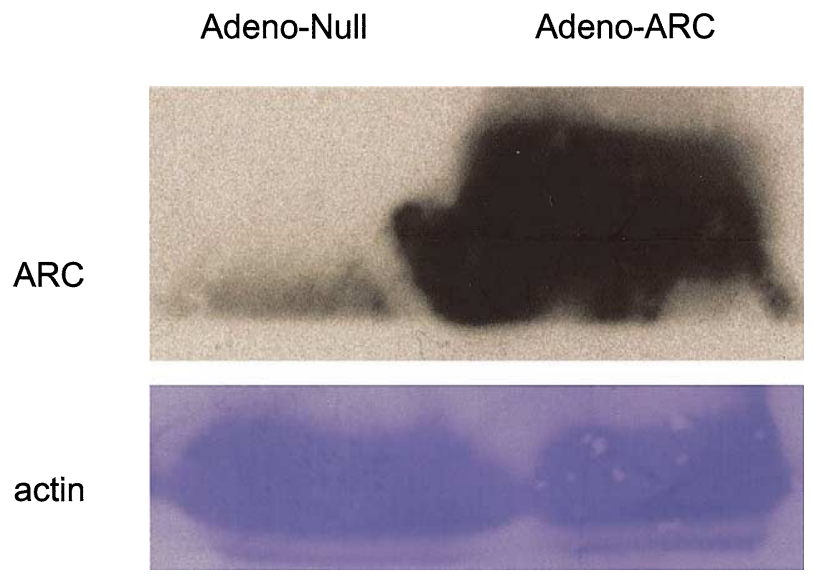

Figure 2. Immunoblotting. Rabbit anti-human polyclonal ARC was used to probe samples at 6 weeks after infarction. There is significant increase in ARC expression in the Adeno-ARC-treated group compared with Adeno-null controls. Samples were also stained for actin to demonstrate equivalent loading conditions.

the LV cavity. The control group received $1000 \mu \mathrm{L}$ of $5.0 \times 10^{10}$ particle-forming units of Adeno-null $(n=7)$ empty vector. After 30 seconds of complete outflow occlusion, the tourniquets were removed, the chest was closed, and the animal recovered without operative intervention for 6 weeks. During this interval, the animal was studied with transthoracic echocardiography at 2-week intervals, and there was 1 operative death $(1 / 16=6.3 \%$ mortality rate $)$. An additional group of 5 native rabbits were used to demonstrate normal measurements of LV ejection fraction and ventricular geometry.

After 6 weeks the animal was returned for nonsurvival surgery. A repeat left thoracotomy exposed the LV free wall. Four 1-mm piezoelectric sonomicrocrystals (Sonometrics Corp, London, Ontario, Canada) were implanted into the myocardium to measure fractional shortening. After sonomicrometry, the heart was arrested in diastole with $1 \mathrm{~mL} \mathrm{KCl}(1 \mathrm{mEq} / \mathrm{mL})$, and the right atrium was incised to allow drainage of blood. The heart was procured and the LV cavity filled with O.C.T. embedding compound (Tissue-Tek O.C.T. or optimal cutting compound; Sakura Finetek, Torrance, Calif) retrogradely through the transected aortic root with a gravity driven pressure reservoir to ensure constant intracavitary pressure. The catheter was removed, the aortic root was ligated, and the heart was placed in a container of O.C.T. embedding compound, bathed in isopentane, frozen in liquid nitrogen, and stored in a freezer at $-80^{\circ} \mathrm{C}$.

\section{Functional Analysis}

Functional analysis was studied by transthoracic echocardiography and sonomicrometry, a well-established technique measuring LV segmental length changes during each cardiac cycle to measure regional contractility. Data were analyzed with Sonoview software (Sonometrics Corp, London, Ontario, Canada) and reported as a percentage of normal contractility \pm SEM. Two sonomicrocrystals were placed within the ischemic segment ("in" position) $10 \mathrm{~mm}$ apart from each other, and 2 sonomicrocrystals within the viable myocardium outside the infarct zone ("out" position) $10 \mathrm{~mm}$ apart. 


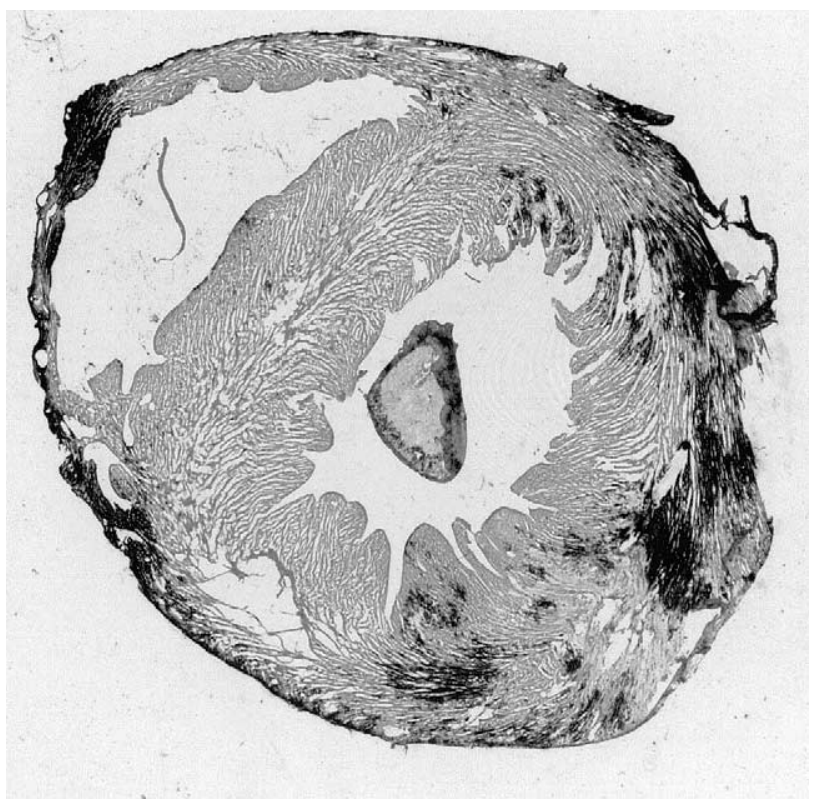

Figure 3. Gene transfection. Demonstration of transfection efficiency by global delivery with great vessel occlusion. Staining for $\beta$-Gal demonstrates that transgene is preferably taken up in $B Z$ region after $\mathbf{3 0}$ minutes of ischemia/reperfusion with transfection of approximately $30 \%$ to $35 \%$ of myocardium.

Fractional shortening is defined as the difference between the end-diastolic length and the end-systolic length divided by the end-diastolic length (the distance between the crystals). Contractility across the BZ was measured by assessing segmental length changes between a crystal in the "in" position with the ipsilateral (in relation to the tourniquet site of occlusion) "out" crystal $10 \mathrm{~mm}$ apart. Normal contractility was measured between the 2 "out" crystals.

\section{Ventricular Geometry: Chamber Size and Wall Thickness}

Four adjacent sections, midway between the base and the apex, spanning the BZ and perpendicular to the LV longitudinal axis, were obtained from each heart to measure chamber luminal diameter and free wall thickness. Four investigators, blinded to the treatment groups, analyzed each section. For the measurement of chamber size, LV diameter was recorded in both the vertical and horizontal axes, and mean values of each slice were averaged. Five equally spaced measurements of the LV free wall from each slice were used to determine wall thickness with an ocular dissecting microscope accurate to $0.03 \mathrm{~mm}$. Five native rabbits were used to demonstrate chamber diameter and wall thickness in uninfarcted and untreated animals.

\section{TUNEL Assay}

Specimens were obtained from storage in a freezer at $-80^{\circ} \mathrm{C}$ and allowed to warm to approximately $-22^{\circ} \mathrm{C}$ in the cryostat. Four adjacent sections, midway between the base and the apex, spanning the BZ were prepared to measure apoptosis. TUNEL assay
(TdT-mediated dUTP nick-end labeling) was performed with a TdT-FragEL DNA Fragmentation Detection Kit (Oncogene Research Products, Boston, Mass). The apoptotic index was taken as a percentage of total apoptotic nuclei per high-powered field examined. Five measurements were obtained from the free wall of each section, by each of two investigators, blinded to the treatment group. The results are reported as the apoptotic indices for each heart \pm SEM.

\section{Immunoblotting}

Specimens obtained for Western blotting were snap-frozen in liquid nitrogen after removal from the animal, without O.C.T. fixative. Specimens were pulverized, homogenized in 10 volume of sodium dodecylsulfate (SDS) lysis buffer $(100 \mathrm{mmol} / \mathrm{L}$ Tris, $\mathrm{pH}$ 8.0, $10 \% \mathrm{SDS}, 10 \mathrm{mmol} / \mathrm{L}$ ethylenediamine tetraacetic acid, 50 $\mathrm{mmol} / \mathrm{L}$ dithiothreitol (DTT), and sheared with a 25 -gauge needle. Samples were normalized for total protein content, and $50 \mu \mathrm{g}$ of each sample were electrophoresed on a $12.5 \%$ SDS-polyacrylamide gel after the addition of 6X sample loading buffer and 3 minutes of denaturation at $100^{\circ} \mathrm{C}$. Proteins were then transferred to Immobilon-P (Millipore, Bedford, Mass) with a wet transfer apparatus. The membrane was subsequently blocked with $5 \%$ nonfat dry milk in Tris-buffered saline solution containing $0.05 \%$ Tween 20. Immunoblotting was performed with a polyclonal antibody against human $\mathrm{ARC}$ at a 1:100 dilution. ${ }^{22}$ Detection was performed with the ECL kit (Amersham, Piscataway, NJ).

\section{Statistical Analysis}

All data are expressed as mean value \pm SEM. Statistical analyses were performed with INSTAT (GraphPad Software, Sorrento Valley, Calif). Comparisons between 2 groups were analyzed by unpaired Student's $t$ test. Multiple comparisons against the control were first analyzed by 1-way analysis of variance followed by the Tukey-Kramer modified unpaired Student's $t$ test to determine significance between groups.

\section{Results \\ Demonstration of Global Delivery and Transgene Expression}

An adenoviral construct containing the $\beta$-Gal reporter gene was used with this technique of viral delivery demonstrating transfection of approximately $30 \%$ to $35 \%$ of myocardium preferentially in the BZ and LV free wall (Figure 3). Immunoblotting demonstrated increased expression of ARC at the $\mathrm{BZ}$ in the Adeno-ARC treated group compared with the controls at 6 weeks as seen in Figure 2.

\section{Improvement of LV Function After Adeno-ARC Treatment}

Baseline ejection fraction for a group of native rabbits $(\mathrm{n}=$ 5 ) before surgery was $42 \% \pm 1.2 \%$. Two weeks after ischemia, there was no difference in ejection fraction between the Adeno-ARC (A) and the control (C) groups (A = $35 \% \pm 3.1 \%$ and $\mathrm{C}=32 \% \pm 1.5 \% ; P=.41$. However, ARC rabbits demonstrated greater preservation of function than did at 4 weeks $(\mathrm{A}=38 \% \pm 1.9 \%$ and $\mathrm{N}=29 \% \pm$ 


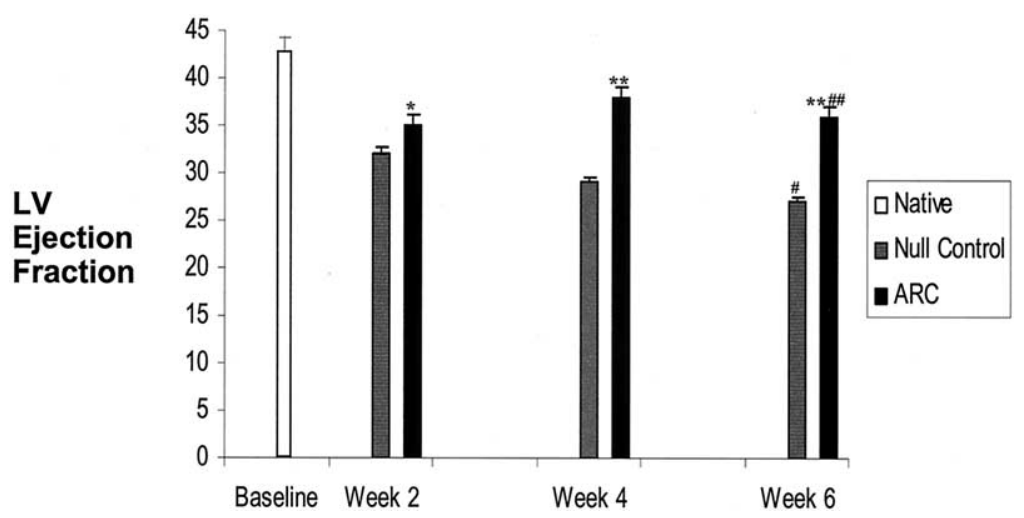

Figure 4. Echocardiography. Global myocardial function measured by LV ejection fraction from transthoracic echocardiography. At 2 weeks, there is no significant difference among the 3 groups. However, ARC-treated animals maintained higher EF at weeks 4 and 6 compared with the Adeno-null controls. For ARC-treated animals compared with controls at the same time point: ${ }^{*} P=N S$; ${ }^{* *} P<.001$. Comparison of control week 2 to control week $6\left({ }^{\#} P<.01\right)$, and comparison of ARC week 2 to ARC week $6\left({ }^{\# \# P}=N S\right)$.

$2.0 \% ; P<.001)$ and at 6 weeks $(\mathrm{A}=36 \% \pm 3.1 \%, \mathrm{C}=$ $27 \% \pm 1.8 \% ; P<.001)$. In addition, whereas the control rabbits had a continued decline in function between weeks 2 and $6(P<.01)$, the ARC group maintained function between weeks 2 and $6(P=.80)$. Although the ARC group was superior to the control group, it was still less than the native group at each time point $(P<.01)$ (Figure 4$)$. Preservation of fractional shortening in the ARC group at 6 weeks was significantly greater than that in the control group $(\mathrm{A}=70.81 \% \pm 8.79 \%$ and $\mathrm{C}=49.76 \% \pm 2.22 \%$; $P<.048)$ (Figure 5).

\section{Preservation of Ventricular Geometry}

Compared with native rabbits (uninfarcted and untreated), the Adeno-ARC group demonstrated superior preservation of ventricular geometry and wall thickness than the controls. Rabbits treated with gene transfer of ARC had a mean chamber diameter of $13.59 \pm 0.47 \mathrm{~mm}$ (native $[\mathrm{N}]=$ $13.44 \pm 0.56 \mathrm{~mm}, P=.84)$ and free wall thickness of $3.48 \pm 0.18 \mathrm{~mm}(\mathrm{~N}=3.61 \pm 0.10 \mathrm{~mm}, P=.54)$. Control rabbits had a significant increase in mean $\mathrm{LV}$ mid-chamber diameter $(\mathrm{C}=16.87 \pm 0.88 \mathrm{~mm} ; P<.01$ compared with both native and Adeno-ARC) and loss of wall thickness $(\mathrm{C}=2.57 \pm 0.15 \mathrm{~mm} ; P<.01$ compared with both native and Adeno-ARC). The results are summarized in Figures 6 and 7. A representative sample from each of the 3 groups is seen in Figure 8.

\section{Demonstration of Apoptosis}

The number of apoptotic cells was expressed as a percentage of total nuclei per high-powered field examined. A significant reduction in apoptosis in the ARC-treated group $(0.77 \% \pm 0.16 \% ; P<.0001)$ compared with the controls
$(2.77 \% \pm 0.35 \%)$ was observed (Figure 9). Representative sections are depicted in Figure 10.

\section{Conclusions}

This study demonstrates that adenoviral gene therapy with ARC can block apoptosis after myocardial ischemia to preserve ventricular function and geometry over a 6-week course in a rabbit model. This is the first in vivo study to use Adeno-ARC.

Our method of viral gene delivery with aortic and pulmonary artery occlusion resulted in transfection of $30 \%$ to $35 \%$ of the myocardium, preferentially in the BZ and LV free wall. The advantage to occlusion of the PA before aortic occlusion is the opportunity for the left atrium and ventricle to empty lowering LV end-diastolic volume reducing LV end-diastolic pressure. As a result, intracavitary injection of the viral vector with both aortic and PA occlusion allows greater diffusion into the coronary arteries and the myocardial capillary bed because the reduced LV enddiastolic pressure creates a more favorable gradient for the virus to transfect the myocardium. In addition, there is less hemodynamic dysfunction by avoiding a more distended left ventricle beating against an occluded aorta. The preferential localization into the BZ may be due to alterations in endothelial cell permeability in this region after ischemia. In addition, transgene expression of ARC was elevated in the Adeno-ARC group 6 weeks after delivery, demonstrating feasibility of delivery.

We expect that the administration of ARC after the immediate ischemia/reperfusion insult would not confer a protective benefit for at least 1 to 2 days because that would be the minimal amount of time required for viral gene expression to first be detected. At 2 weeks both groups of 


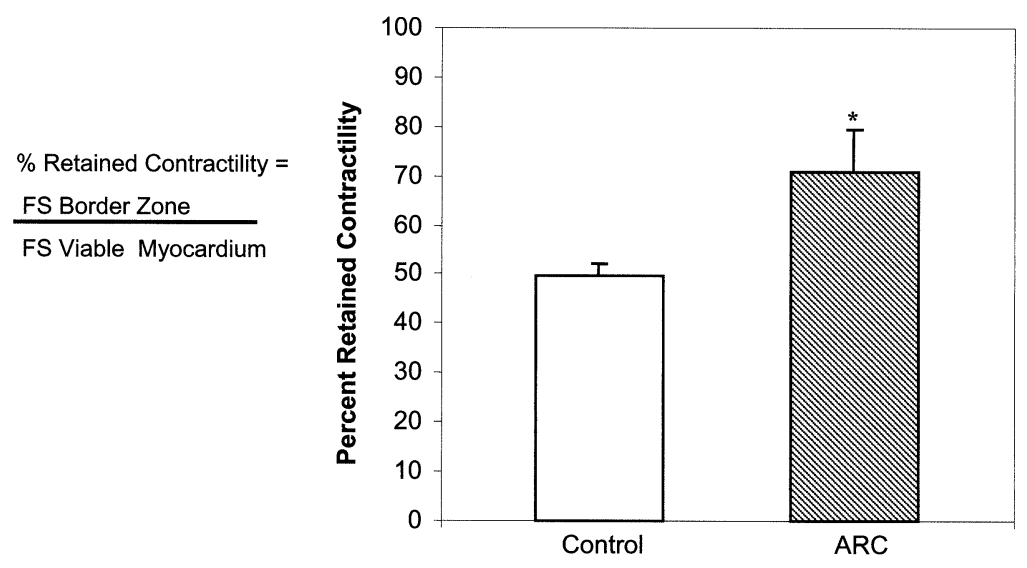

Figure 5. Sonomicrometry. There is superior preservation of fractional shortening (FS) across BZ in the ARC group at 6 weeks compared with the Adeno-null controls. Results are reported as a percentage of baseline FS of BZ compared with remote viable myocardium. ( ${ }^{*} P<.05$ for ARC compared with controls.)

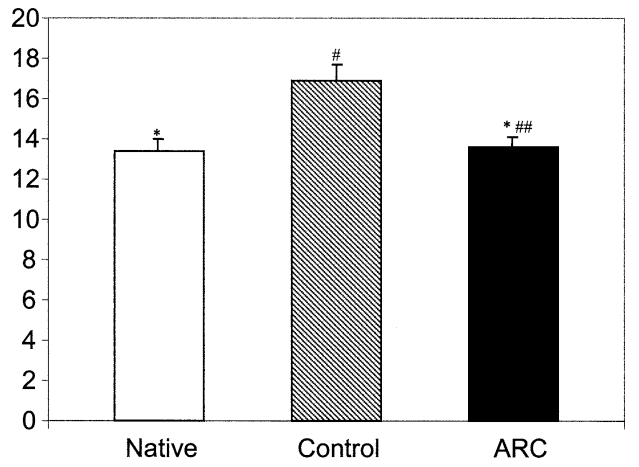

Figure 6. Assessment of LV chamber size. LV dilation was measured by ventricular diameter at the $B Z$. $\left({ }^{*} P<.01\right.$ when compared with Adeno-null control group. ${ }^{\#} \boldsymbol{P}=\mathrm{NS}$ and ${ }^{\# \#} \boldsymbol{P}<.01$ when compared with native group.)

animals experience a decline in function caused by the infarction as seen by echocardiography. However, by 4 weeks the benefit in the ARC group is apparent and continues at 6 weeks. Importantly, despite the initial decline in $\mathrm{EF}$ after infarction seen at 2 weeks, there was no continued decline in the ARC group as opposed to the continuous deterioration in function seen in the controls. This suggests that ARC can prevent a decline in function.

By 6 weeks, regional function with sonomicrometry demonstrates greater preservation of contractility across the $\mathrm{BZ}$ in ARC animals. The ratio of preservation of fractional shortening across the $\mathrm{BZ}$ compared with the viable myocardium serves as a control against the contractile state of the heart. LV function after infarction is first maintained by hypertrophy in the BZ region, and then the remaining myocardium is recruited as needed to hypertrophy in an effort to maintain stroke volume. The transition to decompensated hypertrophy is characterized by a decline in contractility

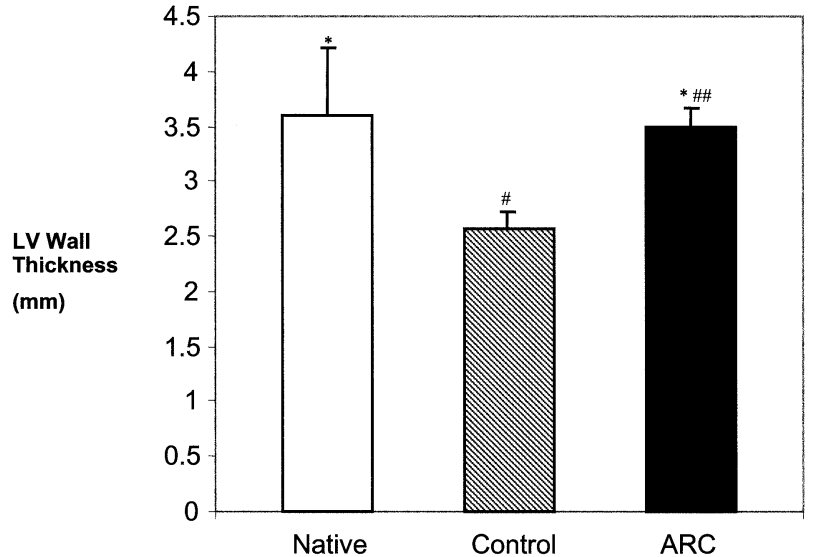

Figure 7. Assessment of LV free wall thinning. LV free wall thinning at $B Z$ was measured by LV free wall thickness. ( ${ }^{*} P<.01$ when compared with Adeno-null control group. ${ }^{\#} \boldsymbol{P}=\mathrm{NS}$ and ${ }^{\# \#} \boldsymbol{P}<.01$ when compared with native group.)

across the BZ followed by further hypertrophy in the remaining myocardium until the remaining myocardium can no longer compensate and heart failure ensues. This analysis, as a result, allowed us to determine the specific contractility of the $\mathrm{BZ}$ with respect to the remaining myocardium. Most important, the correlation of the preservation of fractional shortening with echocardiography served as a validation for both techniques of analysis in this rabbit model.

In addition to a significant number of myocytes undergoing apoptosis in the ischemic segment during the initial period of ischemia/reperfusion injury, it appears that apoptosis is present at the BZ beyond the initial injury. Initial myocyte loss may be coupled with additional cell loss over time due to apoptosis. Although the TUNEL assay is not 


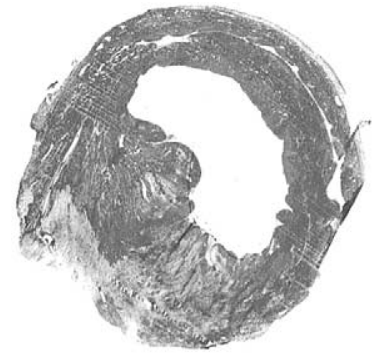

Native Heart

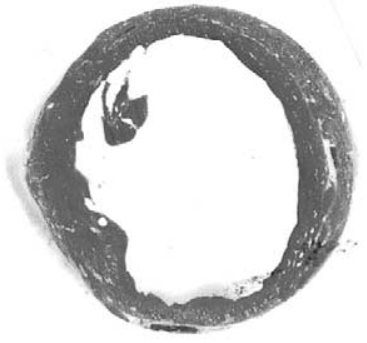

Null Control Infarcted Heart

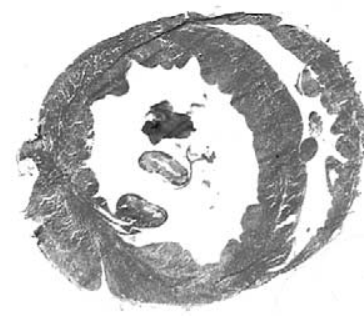

ARC Treated Infarcted Heart

Figure 8. Representative sections at BZ. Representative mid chamber sections are depicted above. Left, Native (uninfarcted and untreated); middle, Adeno-null control; right, Adeno-ARC. ARC-treated group preserved midchamber transverse diameter and LV free wall thickness to a greater extent when compared with natives than the control group.

specific for cardiomyocytes, recent reports have determined that nearly all apoptotic bodies are cardiomyocytes. ${ }^{18,19}$ Olivetti noted that the mechanism of ventricular wall thinning of the remaining myocardium was the result of a $36 \%$ reduction in the mural number of myocytes over time. ${ }^{1}$ This cell loss within the myocardium may further destabilize the ventricular scaffolding resulting in cell-cell slippage of the remaining myocytes. ${ }^{3}$ Thus, the lateral slippage of myocytes may condition the onset, development, and progression of heart failure that evolves after an acute myocardial infarction. ${ }^{20}$

This study suggests that apoptosis exacerbates myocardial cell death after infarction resulting in progressive ventricular dilation. The trigger for apoptosis over the chronic period may be due to the abnormal passive stretching produced by bulging of the necrotic infarct region during ventricular contraction across the BZ. ${ }^{21}$ It appears that progressive stretch leads to an increase in cytosolic calcium levels destabilizing the mitochondrial membrane and triggering the release of cytochrome-c and apoptosis. ${ }^{22}$ As the mechanical disadvantage of higher wall tension is augmented with infarct maturation, ventricular stiffening, and progressive chamber dilation, the continued stretch across the BZ may initiate and sustain further apoptosis through multiple mechanisms. Thus, the benefit over the course of 6 weeks in the ARC-treated group would likely be due to an anti-apoptotic effect that occurs after the initial ischemia/ reperfusion injury.

Multiple theories exist to explain global LV dysfunction with respect to dysfunction of the BZ, a region of normally perfused but poorly functioning myocardium. ${ }^{23}$ Increased wall stress in the $\mathrm{BZ}$ after myocardial infarction has been demonstrated as a result of mechanical tethering of the $\mathrm{BZ}$ region to adjacent noncontracting myocardium. ${ }^{24,25}$ This has also been shown to lead to increased apoptosis in the BZ. In addition, pathologic alterations in the BZ may occur be-

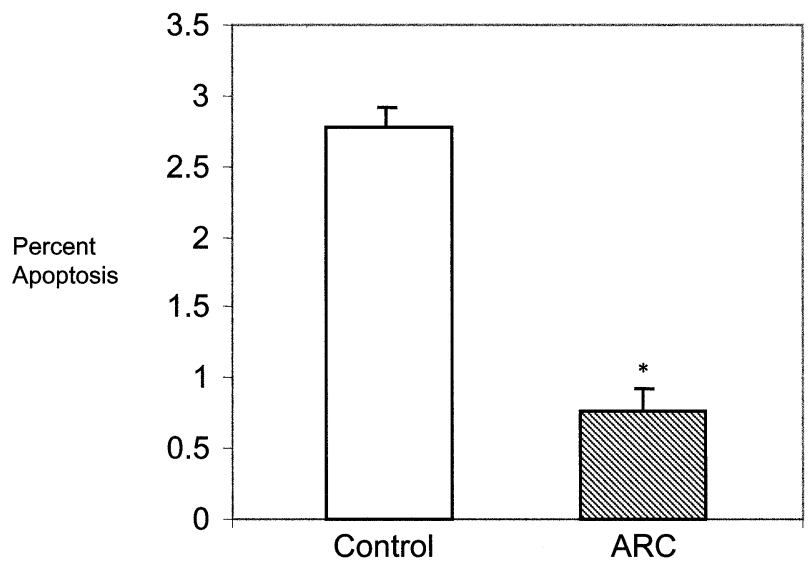

Figure 9. Apoptotic index. A significant reduction in the percentage of apoptotic cells in the ARC group compared with the controls after 6 weeks is demonstrated. ( ${ }^{*} P<.0001$ when compared with control rabbits.)

cause the oxygenation potential of the surviving myocardium is significantly reduced in this region, making the cells more susceptible to additional ischemic episodes. ${ }^{26}$ Thus, $\mathrm{BZ}$ dysfunction is likely multifactorial after ischemia.

There was a significant reduction in the degree of apoptosis in the ARC-treated group $(0.77 \% \pm 0.15 \%)$ compared with the controls $(2.77 \% \pm 0.35 \%)$. Although there is wide variability in terms of the extent of apoptosis reported after myocardial ischemia, these data are consistent with other studies. Olivetti and associates ${ }^{26}$ noted $12 \%$ of myocytes in the BZ and $1 \%$ of myocytes in the remote myocardium showed apoptosis in patients who died within 10 days after myocardial infarction. Sharov and coworkers ${ }^{27}$ noted $4 \%$ in the $\mathrm{BZ}$ and less than $0.5 \%$ in the remote myocardium in a dog model of infarction and subsequent heart failure. Overexpression of ARC appears to have conferred signifi- 


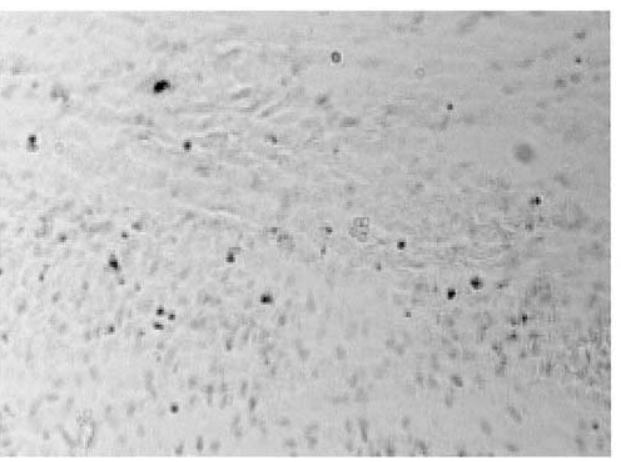

Null Control

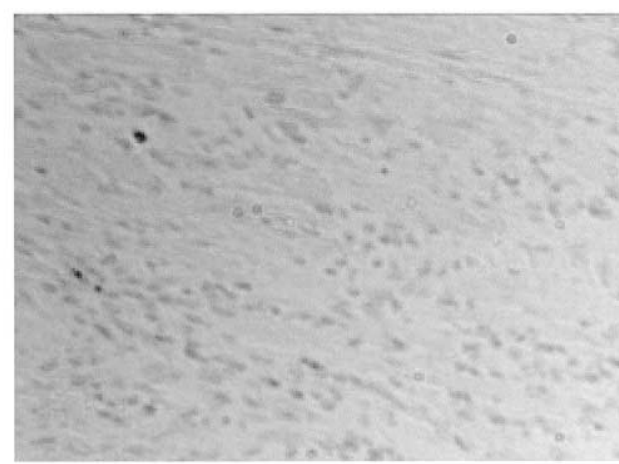

Adeno-ARC

\section{Figure 10. Representative TUNEL sections. Samples from BZ region from control sample and ARC-treated sample demonstrate significant reduction in darkly staining apoptotic bodies in the ARC group.}

cant protection from apoptosis during the entire 6 weeks examined whereas gene expression was maintained. It would be important to determine whether this protective effect of ARC would still be present over a longer period once the gene was no longer expressed. It may be that further long-term protection requires continued ARC expression; on the other hand, it may be that ARC is only required during a critical period soon after the infarction to "rescue" the heart by blocking apoptosis.

We postulate that apoptosis persists within the $\mathrm{BZ}$ region after infarction, which contributes to myocardial stretch and eventual ventricular dilation and dysfunction. The data suggest that blocking apoptosis in this region can prevent pathologic ventricular remodeling. Administration of Adeno-ARC leads to a reduction in apoptosis, preservation of ventricular geometry, and prevention of postischemic heart failure. As the limitations of gene therapy are overcome, its ultimate clinical application as an adjunct to the conventional pharmacologic and interventional management of myocardial infarction may be approaching. This study represents a small, preliminary yet important step to this goal. There are a number of issues that will have to eventually be worked out, such as the ideal vector for delivery, the ideal method of delivery (direct intramyocardial injection vs intracoronary delivery with coronary angiography vs intracavitary delivery with temporary outflow occlusion), and the optimal timing after myocardial infarction during which to attempt delivery, among others. Nevertheless, the potential application of this strategy and clinical benefit is readily apparent.

We are grateful to Timothy Pirolli and Jeffrey Burdick for technical support.

\section{References}

1. Olivetti G, Capasso JM, Sonnenblick EH, Anversa P. Side-to-side slippage of myocytes participates in ventricular wall remodeling acutely after myocardial infarction in rats. Circ Res. 1990;67:23-34.
2. Cheng W, Li B, Kajstura J, Li P, Wolin MS, Sonnenblick EH, et al. Stretch-induced programmed myocyte cell death. J Clin Invest. 1995; 96:2247-59.

3. Anversa P, Ricci R, Olivetti G. Quantitative structural analysis of the myocardium during physiologic growth and induced cardiac hypertrophy: a review. J Am Coll Cardiol. 1986;7:1140-9.

4. Olivetti G, Abbi R, Quaini F, Kajstura J, Cheng W, Nitahara JA, et al. Apoptosis in the failing human heart. N Engl J Med. 1997;336:113141.

5. Olivetti G, Capasso JM, Meggs LG, Sonnenblick EH, Anversa P. Cellular basis of chronic ventricular remodeling after myocardial infarction in rats. Circ Res. 1991;68:856- 9.

6. Saraste A, Pulkki K, Kallajoki M, Heikkila P, Laine P, Mattila S, et al. Cardiomyocyte apoptosis and progression of heart failure to transplantation. Eur J Clin Invest. 1999;29:380-6.

7. Sabbah HN. Apoptotic cell death in heart failure. Cardiovasc Res. 2000;45:704-12.

8. Reed JC. Mechanisms of apoptosis. Am J Pathol. 2000;157:1415-30.

9. Haunstetter A, Izumo S. Apoptosis: basic mechanisms and implications for cardiovascular disease. Circ Res. 1998;82:1111-29.

10. Cohen GM. Caspases: the executioners of apoptosis. Biochem J. 1997;326:1-16.

11. Garcia-Ruiz C, Colell A, Mari M, Morales A, Fernandez-Checa JC. Direct effect of ceramide on the mitochondrial electron transport chain leads to generation of reactive oxygen species: role of mitochondrial glutathione. J Biol Chem. 1997;272:11369-77.

12. Adachi S, Cross AR, Babior BM, Gottlieb RA. Bcl-2 and the outer mitochondrial membrane in the inactivation of cytochrome $\mathrm{c}$ during Fas-mediated apoptosis. J Biol Chem. 1997;272:21878-82.

13. Carswell EA, Old LJ, Kassel RL, Green S, Fiore N, Williamson B. An endotoxin-induced serum factor that causes necrosis of tumors. Proc Natl Acad Sci U S A. 1975;72:3666-70.

14. Koseki T, Inohara N, Chen S, Nunez G. ARC, an inhibitor of apoptosis expressed in skeletal muscle and heart that interacts selectively with caspases. Proc Natl Acad Sci U S A. 1998;95:5156-60.

15. Ekhterae D, Lin Z, Lundberg MS, Crow MT, Brosius FC 3rd, Nunez G. ARC inhibits cytochrome $\mathrm{c}$ release from mitochondria and protects against hypoxia-induced apoptosis in heart-derived H9c2 cells. Circ Res. 1999;85:e70-7.

16. Neuss M, Monticone R, Lundberg MS, Chesley AT, Fleck E, Crow MT. The apoptotic regulatory protein ARC (apoptosis repressor with caspase recruitment domain) prevents oxidant stress-mediated cell death by preserving mitochondrial function. J Biol Chem. 2001;276: 33915-22.

17. Hersh J, Crystal RG, Bewig B. Modulation of gene expression after replication-deficient recombinant adenovirus-mediated gene transfer by the product of a second adenovirus vector. Gene Ther. 1995;2:12431. 
18. Takemura G, Ohno M, Hayakawa Y. Role of apoptosis in the disappearance of infiltrated and proliferated interstitial cells after myocardial infarction. Circ Res. 1998;82:1130-8.

19. Gottlieb RA, Burleson KO, Kloner RA, Babior BM, Engler RL. Reperfusion injury induces apoptosis in rabbit cardiomyocytes. J Clin Invest. 1994;94:1621-8.

20. Fliss H, Gattinger D. Apoptosis in ischemic and reperfused rat myocardium. Circ Res. 1996;79:949-56.

21. Page E, Polimein PI. Ultrastructural changes in the ischemic zone bordering experimental infarcts in rat left ventricles. Am J Pathol. 1977;87:81-104

22. Pan J, Fukuda K, Saito M, Matsuzaki J, Kodama H, Sano M, et al. Mechanical stretch activates the JAK/STAT pathway in rat cardiomyocytes. Circ Res. 1999;84:1127-36.

23. Moulton MJ, Downing SW, Creswell LL, Fishman DS, Amsterdam DM, Szabo BA, et al. Mechanical dysfunction in the border zone of an ovine model of left ventricular aneurysm. Ann Thorac Surg. 1995;60: 986-97.

24. Kramer CM, Lima JA, Reichek N, Ferrari VA, Llaneras MR, Palmon LC, et al. Regional differences in function within noninfarcted myocardium during left ventricular remodeling. Circulation. 1993;88: 1279-88.

25. Wyatt HL, Forrester JS, da Luz PL, Diamond GA, Chagrasulis R, Swan HJ. Functional abnormalities in nonoccluded regions of myocardium after experimental coronary occlusion. Am J Cardiol. 1976; 37:366-72.

26. Olivetti G, Ricci R, Beghi C. Response of the borderzone myocardial infarction in rats. Am J Pathol. 1986;125:476-83.

27. Sharov VG, Sabbah HN, Shimoyama H, Goussev AV, Lesch M, Goldstein S. Evidence of cardiocyte apoptosis in myocardium of dogs with chronic heart failure. Am J Pathol. 1996;148:141-9.

\section{Discussion}

Dr G. Alexander Patterson (St Louis, Mo). The distribution of the transfection was not entirely clear to me. You showed a very nice slide of the distribution of $\beta$-Gal, and it struck me that most of the distribution was on the epicardium, not within the cardiac muscle itself. It seemed to me a very superficial distribution. Do you have any immunohistochemistry or some other assay that would allow us to know the distribution of the Adeno-ARC? Is it the same as that of $\beta$-Gal?

Dr Chatterjee. The $\beta$-Gal distribution is both within the epicardium and also within most of the myocardium. In terms of Adeno-ARC expression, what we relied on was immunoblotting at 6 weeks after injection. This showed that there was a significant increase, about a 20 -fold increase in intensity, within the BZ region of the heart.

Interestingly, when we compared Adeno-ARC expression in the viable normal myocardium outside the infarct region, there was only a very small increase in expression in the ARC-treated group compared with the null controls. So, according to our immunoblotting at 6 weeks, there appears to be preferential localization of Adeno-ARC within the $\mathrm{BZ}$ region as opposed to other regions of the heart.

Dr Christopher A. Caldarone (Iowa City, Iowa). I was interested in the mechanism of ARC in the myocyte. You pointed out that it worked at the level of caspase 8 and preventing release of cytochrome $\mathrm{C}$, presumably resulting in less caspase activity downstream. Did you make any attempt to measure caspase activity in these specimens? The other question was in reference to the apoptotic index.

Dr Chatterjee. In terms of measuring caspase activity, that is a very good point. The next step in this study is looking at caspase 3 activity, as well as caspase 8 levels within our treated samples to determine how much of a reduction occurs. In vitro evidence demonstrates that in cell lines, injecting Adeno-ARC can block both caspase 8 in the extrinsic pathway of apoptosis, as well as the release of cytochrome $\mathrm{C}$ from the mitochondria in the intrinsic pathway. Much of this is believed to be mediated by preventing reactive oxygen species activity that results from ischemic injury.

In terms of your second question, the apoptotic index was the percent of cells per high-powered field that were apoptotic. In the control group it was about $2.7 \%$, and in the ARC-treated group it was $0.7 \%$, a 4 -fold reduction.

Dr Caldarone. The reason that first question might be pertinent is because a recent study in human dilated ischemic cardiomyopathy demonstrated apoptosis occurring through caspase-independent mechanisms. Therefore, I believe measuring the caspase activity would be important.

Dr Chatterjee. Certainly. What is interesting with ARC is that ARC has been shown to work not only in the caspase-dependent, the death receptor-mediated pathway mediated by tumor necrosis factor receptor 1 , but also through the caspase-independent pathway, as you mentioned, through cytochrome c. The benefit of ARC, in theory, is that it acts on both arms of the apoptotic cascade, and certainly in vitro evidence demonstrates a significant reduction in downstream caspase 3 activity, the final common pathway of apoptosis.

Dr Ralph J. Damiano, Jr (St Louis, Mo). One possibility, and you mentioned it in your introduction, is that ARC may also have a beneficial effect on preventing necrosis. Did you look at infarct size in these animals, particularly in the area at risk? Was there any effect on cell necrosis as contrasted to its primary effect on apoptosis?

Dr Chatterjee. That is an excellent point. We have actually looked at other anti-apoptotic agents in a similar model, and in those groups we have seen no real change in the size of the infarct. Our theory is that the size of the infarct is largely determined right away in the first 24 hours; however, it really takes about 48 to 72 hours before transgene expression begins and ARC can exert a protective effect. The acute infarct size is probably determined before ARC is even expressed. What we do see is a small increase in fibrosis within the BZ of the null group, suggesting that there may be, in addition to blocking apoptosis, some antinecrosis benefit within the $\mathrm{BZ}$ region of the ARC-treated group. 